\title{
High-frequency ultrasound in ex vivo animal lungs in pulmonary edema
}

\author{
Jacek A. Wojtczak ${ }^{1 *}$ and Ronald W. Wood ${ }^{2}$ \\ *Correspondence: gryf70@yahoo.com \\ 'Department of Anesthesiology, University of Rochester School of Medicine and Dentistry, Rochester, New York, USA. \\ ${ }^{2}$ Department of Obstetrics/Gynecology, Urology, and Neurobiology and Anatomy, University of Rochester School of Medicine and \\ Dentistry, Rochester, New York, USA.
}

\begin{abstract}
Background: The purpose of this study was to determine if ex vivo animal lungs could provide a method to investigate the etiology of ultrasonic B-lines ("lung comets"). B-lines are acoustic artifacts associated with the accumulation of extravascular lung water.

Methods: Transthoracic and epipleural sonograms were recorded in the endotracheally-intubated post mortem lapine and porcine lung specimens. Ultrasound imaging was performed using $6 \mathrm{MHz}$ curved, $7.5 \mathrm{MHz}$ or $14 \mathrm{MHz}$ linear and $40 \mathrm{MHz}$ transducers. To visualize subpleural gross pathomorphology, deep longitudinal cuts were made in the same areas of the lung that had been examined sonographically. Macroscopic digital photographs were obtained using gross specimen photography system.

Results: B-lines were detected within one hour after the animal's death and became dense and confluent in many areas within 2 to 6 hours. Florid pulmonary edema was evidenced by large amounts of pink frothy fluid in endotracheal tubes. Macroscopic crosssections revealed a subpleural mosaic of punctate red/purple hemorrhagic "microlesions" in the areas where B-lines were detected. Epipleural high frequency (40 MHz), three-dimensional (3D) ultrasound displayed B-lines as very dense, vertical, "stalactite"- like structures trailing down from the pleural surface. In the horizontal (transverse) plane B-line cross sections had oval shapes which were approximated by the Image J computational program to an ellipse with the major and the minor axes. The mean diameter of fifty two B-lines in one selected slice was $0.54 \pm 0.2 \mathrm{~mm}$ (major axis) and $0.25 \pm 0.08 \mathrm{~mm}$ (minor axis).

Conclusions: Ex vivo lung is a convenient and affordable model to study the mechanisms of B-line generation. The ex vivo model is stable enough to allow serial sonograms and monitoring of the lung epipleural and subpleural pathomorphology over several hours after the animal's death. High frequency 3D sonography may be a technology of choice to elucidate the etiology of B-lines.
\end{abstract}

Keywords: Lung ultrasound, B-lines, extravascular lung water, pulmonary edema, high-frequency three-dimensional ultrasound

\section{Introduction}

The normal lung surface is a specular (i.e. mirror-like) reflector of ultrasound. Partially aerated lungs transmit ultrasound through fluid-filled alveoli into the enclosed spaces surrounded by air-filled alveoli [1-3]. The beam is reflected between these acoustically mismatched interfaces. Each reflection is transmitted to the transducer and displayed superimposed on previous reflections resulting in an image composed of all the reflections, i.e. a reverberation artifact $[2,3]$. Some of the reverberation artifacts in the lung may present as vertical, narrow bands extending down from the visceral pleura $[1,4]$. They are called sonographic B-lines. The name "ultrasound lung comets" or "comet-tail artifacts" has been used in the literature before the consensus conference on lung ultrasound [5] introduced a uniform nomenclature. B-lines can range from rare to confluent and finally to a full echogenic lung, or white lung $[4,6-8]$. Several studies showed a tight correlation between B-lines and extravascular lung water accumulation resulting in interstitial or interstitial-alveolar syndrome [1,4,7-10].

Extravascular lung water content was shown to predict survival in critically ill patients [11]. B-lines were also used to distinguish between congestive heart failure and chronic obstructive pulmonary disease [7]. Volpicelli et al., [12] showed that lung ultrasound monitoring and B-line quantification documents clinical improvement of patients with decompensated heart failure at least as well as serial radiologic estimates or levels of natriuretic hormones.

After the initial clinical paper correlating B-line sonograms with radiographic images of diffuse alveolar-interstitial syndrome [4], very few basic studies have been conducted to identify structural components of the lung responsible for the generation of B-lines $[2,3,6,13,14]$. Recently, Soldati et al., [6] showed that B-lines appear in histologically normal ex vivo rabbit lungs that are deflated to a critical level of density which implies that pulmonary acoustic artifacts can be generated independent of any anatomic landmark or other identifiable structures.

Here we further explore the experimental use of ex vivo animal lungs to investigate the mechanisms of B-line generation.

\section{Materials and Methods}

Three rabbits were killed by pentobarbital euthanasia. Seven 


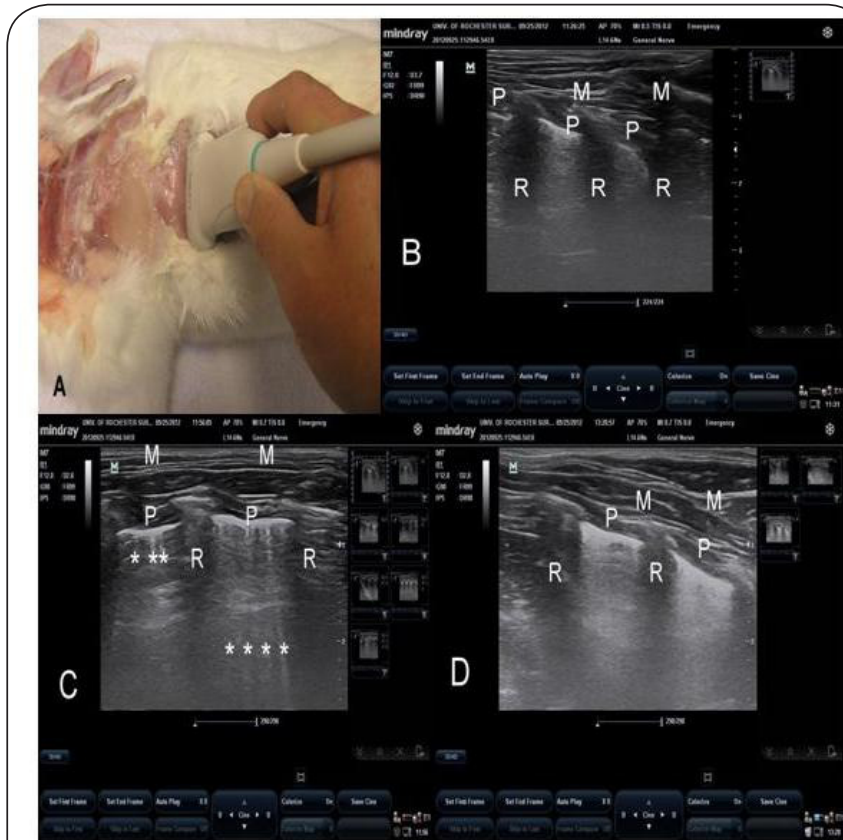

Figure 1. Transthoracic sonograms (14 MHz linear probe) recorded through the muscular layer of the rib cage in a post mortem rabbit (A). Sonograms were performed $15 \mathrm{~min}(\mathrm{~B}), 45 \mathrm{~min}(\mathrm{C})$ and $140 \mathrm{~min}(\mathrm{D})$ after the animal's death. $\mathrm{M}$ - muscles, $\mathrm{R}$ - rib, $\mathrm{P}$ - pleura. B - lines $\left({ }^{*}\right)$ are prominent in the $45 \mathrm{~min}$ sonogram and confluent in the $140 \mathrm{~min}$ sonogram.

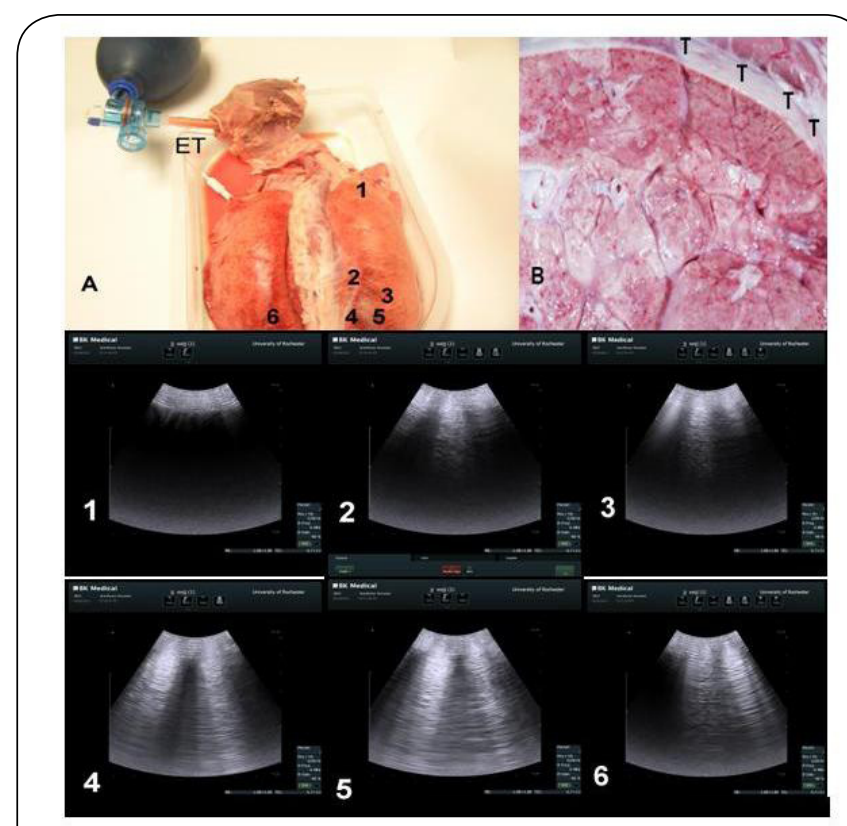

Figure 2. Pig lungs with the intact trachea and larynx examined 2 hours after harvesting. (A). The trachea was intubated and lungs periodically inflated. Immediately after intubation pink frothy fluid appeared in the endotracheal tube (ET). Sonograms $(6 \mathrm{MHz}$ curved probe, Flex Focus, BK Medical) were performed in the well aerated apical lobe (sonogram 1) and in spotty yellow and red/ purple portions of the lung (sonograms 2-6). B-macroscopic exam of the cross section of the same lung showed punctate, hemorrhagic "microlesions". T-position of ultrasound transducers during corresponding sonograms (sonograms 4 and 5). porcine lungs with intact trachea and larynx (Figure 2A) were obtained from a local abattoir at slaughter, packed on ice and transferred within 40 min to the laboratory.

Ultrasound imaging was performed using a $6 \mathrm{MHz}$ curved probe of the Flex Focus 400 system (BK Medical, Peabody, MA), 7.5 MHz linear probe of the CMS 600 system (Contec Medical, China), $14 \mathrm{MHz}$ linear probe of the Mindray M7 system (Mindray Medical, Mahwah, NJ) and $40 \mathrm{MHz}$ probe (704b) of the Visualsonics Vevo 770 system (Toronto, Canada).

Porcine lungs with the intact trachea were intubated immediately after arrival to the laboratory. Lungs were periodically inflated with room air using manual positive pressure ventilation bags. Surface sonograms were recorded directly on the visceral pleura. To visualize subpleural gross pathomorphology, deep longitudinal cuts were made in the same areas of the lung that had been examined sonographically. Macroscopic digital photographs were obtained using gross specimen photography system.

In rabbits ultrasound probes were placed on the muscular layer of the lapine rib cage. Sonograms were performed in 30 min intervals up to 3 hours. After 3 hours thoracotomy was performed, lungs with the trachea excised, and trachea was intubated. Surface sonograms were then performed directly on the visceral pleura.

Data are presented as composite, combining photographs marked with letters and numbers and numbered sonograms. Numbers in the photograph show a position of the ultrasound probe during the recording of a sonogram.

Images obtained with the Visualsonics Vevo 770, high frequency (HF), three dimensional (3D) system were transferred from the ultrasound machine to a desktop computer and analyzed using ImageJ image processing software developed by the National Institute of Health (http://rsb.info.nih.gov/ij/ Accessed August 17, 2010).

\section{Results}

Large amounts of pink frothy fluid suggestive of pulmonary edema appeared in the endotracheal tube in all specimens immediately after intubation (Figure 2A).

A well aerated lungs presented as soft, yellow areas (Figure 2A-1), whereas poorly aerated lungs were red and visibly atelectatic (Figure 4A). Intermediate areas presented as a mosaic of yellow and red/purple portions (Figure 2A-2 to 6).

Sonograms performed in well aerated lungs in closed chest rabbits immediately after death showed muscular layers, lung pleura and a homogenously dark lung image between the ribs (Figure 1B). In well aerated porcine lungs (Figure 2-sonogram 1) a homogenously dark lung image was recorded as the ultrasound beam was reflected from the pleural/air interface.

In closed chest rabbits (Figure 1A), B-lines could be detected within one hour (Figure 1C) after death. B-lines were detected in intermediate areas (spotty yellow and red/purple portions) 


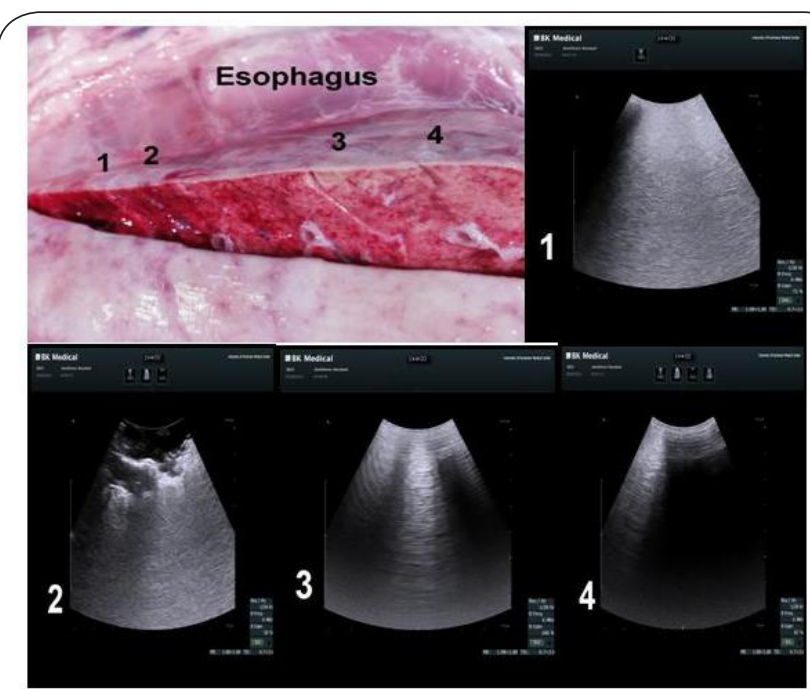

Figure 3. The pig lung specimen examined 6 hours after harvesting. Sonograms were obtained using the curved $6 \mathrm{MHz}$ probe (Flex Focus 400 , BK Medical). The photograph shows the cross section with a confluent hemorrhagic consolidation on the left, corresponding with a sonographic white lung (sonogram 1 and 2). Punctate hemorrhagic "microlesions" on the right side of the specimen correspond with the B-line pattern sonograms (3 and 4$)$.

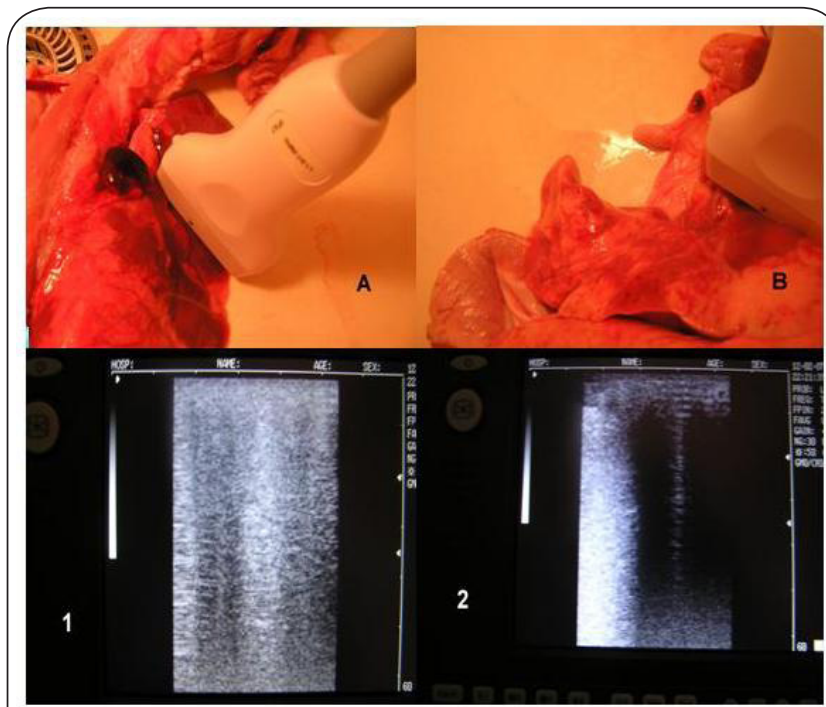

Figure 4. A-totally collapsed apical portion of the lung, the corresponding scan below (1) shows a sonograhic white lung. B-adjacent collapsed (left) and well aerated (right) lung portions. A corresponding sonogram (2) shows a white lung on the left and a black lung (well aerated) with a single B-line on the right side of the image. This porcine specimen was examined with a linear $7.5 \mathrm{MHz}$ transducer (CMS 600, Contec Medical).

of the porcine lung (Figure 2-sonograms 2 to 6).

In both rabbit and porcine lungs B-lines became very dense and confluent in many areas of the lung after about 2 to 6 hours (Figure 1,2 and 3).

A macroscopic cross-sectional exam (Figure 2B) in those areas revealed a diffuse sub-pleural mosaic of punctate red/ purple hemorrhagic "microlesions". Confluent hemorrhagic

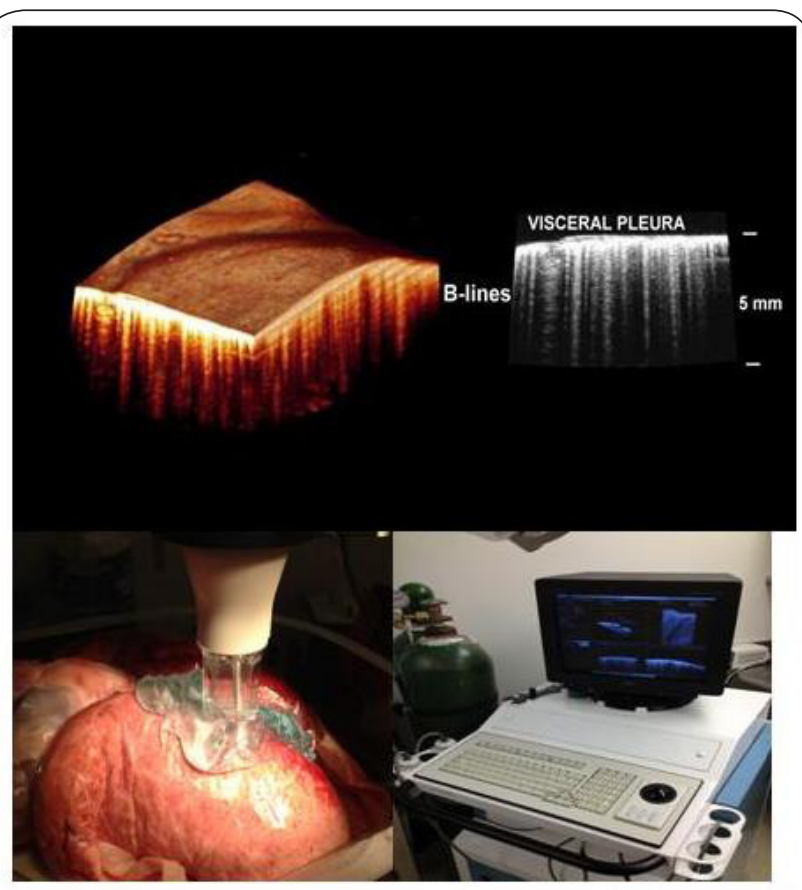

Figure 5. Epipleural high frequency, three-dimensional (3D) ultrasound recordings of B-lines generated in the cortical layer of the porcine lung (upper panel). Recordings were performed using $40 \mathrm{MHz}$ (704b) transducer (lower left) of the Visualsonics Vevo 770 (Toronto, Canada) system (lower right). 3D ultrasound displays B-lines as multiple, vertical, "stalactite" - like structures trailing down from the pleural surface.

A video file (comet_composite-Desktop MP4 video) is accompanying this recording.

consolidations were observed in lungs examined 6 hours after death. Figure 3 shows a specimen of porcine lung with attached esophagus. Numbers mark sites of epipleural sonograms. A sonogram performed in site \# 2 shows subpleural fluid accumulation and a consolidation with an air bronchogram. On the right side less advanced pathological changes were limited to diffuse punctate hemorrhagic "microlesions". Epipleural sonograms in these sites (\# 3 and 4) show a confluent B-pattern.

A variety of ultrasound systems and transducers were used. In general higher frequency linear transducers (Figures 1 and 4) allowed better resolution of B-lines than lower frequency curved transducers (Figure $\mathbf{2}$ and $\mathbf{3}$ ). To further improve resolution and enable accurate quantification of B-lines, high frequency (HF), three-dimensional (3D) sonograms were recorded from the lung surface. HF 3D ultrasound displayed B-lines as very dense, vertical, "stalactite"like structures trailing down from the pleural surface (Figure 5). In the horizontal (transverse) plane B-line cross sections had predominantly oval shapes (Figure 6). Only few B-lines had linear, slit-like shapes (Figure 6).

Oval shapes of B-lines were approximated by the Image J computational program to an ellipse with the major and the minor axes (Figure 7). The mean diameter of fifty two B-lines 


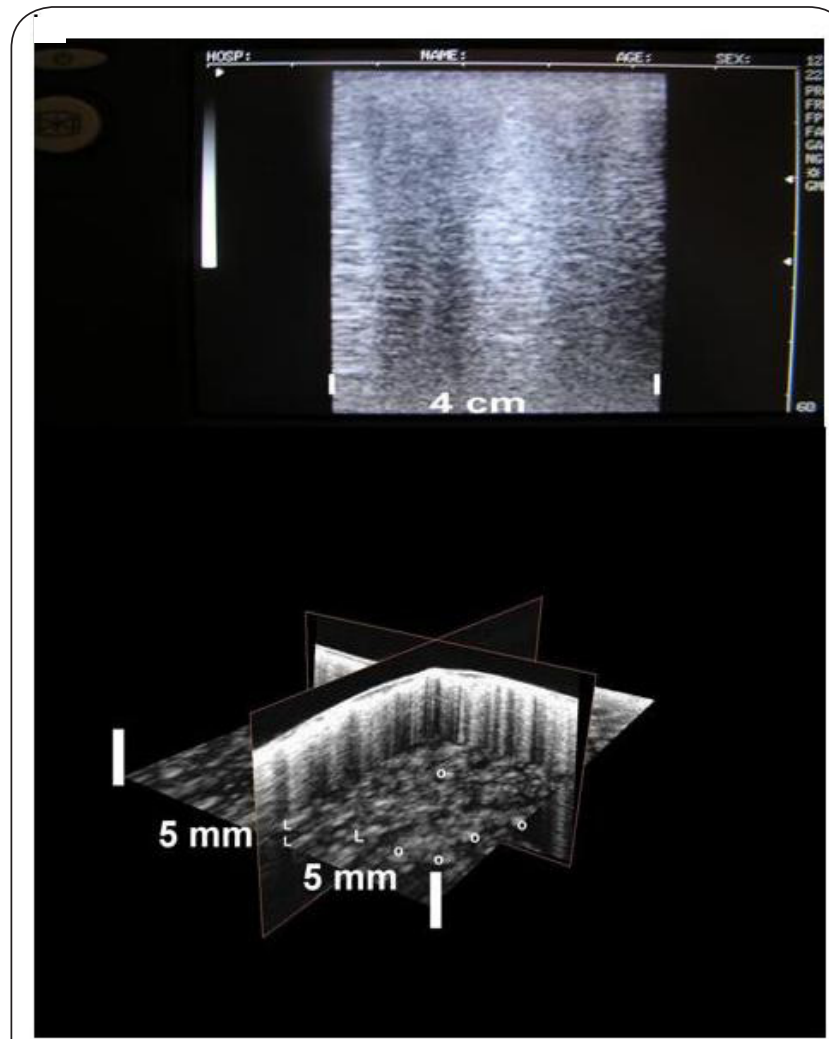

Figure 6. Comparison of a 2D sonogram (upper panel) with a 3D sonogram (lower panel) obtained in the same specimen. The 7.5 MHz linear probe was used to obtain the 2D sonogram and the $40 \mathrm{MHz}$ probe was used to obtain the 3D sonogram. Transverse imaging plane in the lower panel was obtained $2.5 \mathrm{~mm}$ below the surface of the visceral pleura. Cross sections of B-lines have mostly oval $(\mathrm{O})$ shapes. Only few linear (L), slit-like shaped B-lines can be demonstrated.
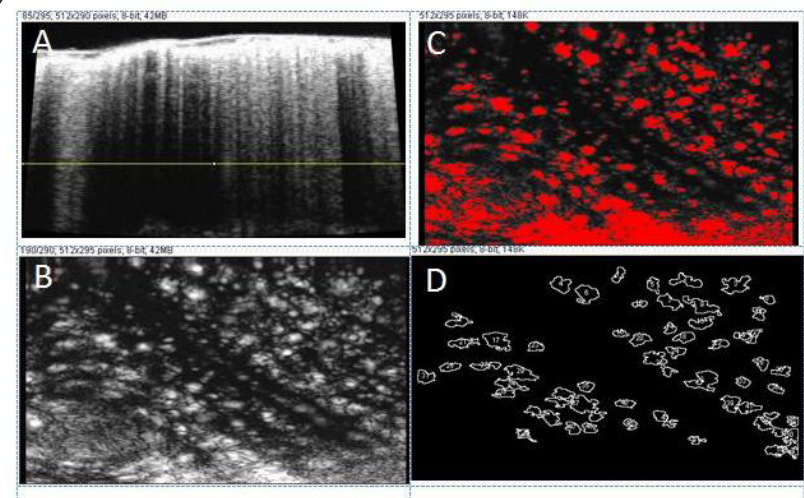

Figure 7. A-B-scan at slice 85. Yellow line shows depth of horizontal plane in panel B. B-horizontal plane.

C-thresholded image before application of size and edge exclusion criteria. D-resultant identified B-line cross-sections with identifying numbers for parameter retrieval. Oval shapes of B-lines (panel B) were approximated to an ellipse with the major and the minor axes. The mean diameter of fifty two B-lines (panel D) in slice 85 was $0.54 \pm 0.2 \mathrm{~mm}$ (major axis) and $0.25 \pm 0.08 \mathrm{~mm}$ (minor axis). in one selected slice was $0.54 \pm 0.2 \mathrm{~mm}$ (major axis) and 0.25 $\pm 0.08 \mathrm{~mm}$ (minor axis).

\section{Discussion}

The injury in ex vivo lungs is caused by "cold ischemia", although lungs are generally resistant to injury [15-17]. Porcine lungs could be transplanted 6 hours after excision [15] and human lungs have been transplanted from cadavers [16]. Since the post mortem changes in the lung progress relatively slowly, the ex vivo model is stable enough to permit serial sonograms and monitoring of lung epipleural and subpleural pathomorphology several hours after the death [17].

This study associated epipleural sonograms with macroscopic tissue images and with underlying lung pathomorphology at the corresponding recording site. Soldati et al., [6] compared rabbit ex vivo epipleural sonograms with histology, however the histological images did not represent the actual recording site. They demonstrated that acoustic artifacts could be reproduced in histologically normal lungs deflated to a density not achievable under physiological conditions. Both approaches suffer from the large size of currently available high frequency linear transducers as shown in Figure 4 and Figure 6 (upper sonogram). Therefore, colocalization of histology with pinpoint changes in the insonated site is difficult if not impossible. Sonographic HF 3D technology enabled us to examine pulmonary acoustic artifacts at higher resolution. The transducer is four times smaller (Figure 5 left lower panel) with much higher resolution than a conventional linear transducer (Figure 6 upper panel). Subpleural B-lines have a "stalactite" type of morphology. The high resolution 3D ultrasound technology enabled transverse images along the horizontal plane across all B-lines in each slice. The B-line cross sections had oval shapes that were approximated during the computational analysis to an ellipse. Lichtenstein et al., [4], first associated B-lines with the radiographic pathology of the interstitial lung syndrome and postulated that B-lines are due to the ultrasound reflection from edematous interlobular septa. This hypothesis has since been reported as fact without any experimental proof. We think it unlikely that densely spaced submilimeter B-line artifacts can be generated by the ultrasound reflection from interlobular septa since septal walls are usually 10 to $25 \mathrm{~mm}$ apart [18] or $7 \mathrm{~mm}$ apart as per radiographic measurements performed by Lichtenstein et al., [4]. It is more likely that B-lines are reflections from discrete air/fluid interfaces between collapsed, fluid-filled and well aerated alveoli. Confirmation of the exact nature of subpleural B-line generators will likely require further investigation combining HF 3D sonography with intravital microscopy.

\section{Conclusions}

Studies of animal lungs ex vivo can improve understanding sonographic artifacts observed during extravascular lung water accumulation in critically ill patients. The ex vivo models 
are sufficiently stable to permit serial sonograms for several hours after the animal's death. HF 3D sonography will likely be useful in further inquiry into the etiology of B-lines.

\section{Additional files}

Supplement video

\section{Competing interests}

The authors declare that they have no competing interests.

\section{Authors' contributions}

Jacek A. Wojtczak and Ronald W. Wood equally contributed in planning the study, collecting and analyzing data and drafting the manuscript.

\section{Publication history}

Received: 28-Jan-2013 Revised: 06-Mar-2013

Accepted: 11-Mar-2013 Published: 24-Apr-2013

\section{References}

1. Volpicelli G: Lung sonography. J Ultrasound Med 2013, 32:165-71. | Article I PubMed

2. Soldati G, Copetti R and Sher S: Sonographic interstitial syndrome: the sound of lung water. J Ultrasound Med 2009, 28:163-74. | Article | PubMed

3. Soldati G, Giunta V, Sher S, Melosi F and Dini C: "Synthetic" comets: a new look at lung sonography. Ultrasound Med Biol 2011, 37:1762-70. | Article | PubMed

4. Lichtenstein D, Meziere G, Biderman P, Gepner A and Barre O: The comet-tail artifact. An ultrasound sign of alveolar-interstitial syndrome. Am J Respir Crit Care Med 1997, 156:1640-6. | Article | PubMed

5. Volpicelli G, Elbarbary M, Blaivas M, Lichtenstein DA, Mathis G, Kirkpatrick AW, Melniker L, Gargani L, Noble VE, Via G, Dean A, Tsung JW, Soldati G, Copetti R, Bouhemad B, Reissig A, Agricola E, Rouby JJ, Arbelot C, Liteplo A, Sargsyan A, Silva F, Hoppmann R, Breitkreutz R, Seibel A, Neri L, Storti E and Petrovic T: International evidence-based recommendations for point-of-care lung ultrasound. Intensive Care Med 2012, 38:577-91. | Article | PubMed

6. Soldati G, Inchingolo R, Smargiassi A, Sher S, Nenna R, Inchingolo CD and Valente S: Ex vivo lung sonography: morphologic-ultrasound relationship. Ultrasound Med Biol 2012, 38:1169-79. | Article | PubMed

7. Lichtenstein $D$ and Meziere $G$ : A lung ultrasound sign allowing bedside distinction between pulmonary edema and COPD: the comet-tail artifact. Intensive Care Med 1998, 24:1331-4. | Article | PubMed

8. Jambrik Z, Monti S, Coppola V, Agricola E, Mottola G, Miniati M and Picano $\mathrm{E}$ : Usefulness of ultrasound lung comets as a nonradiologic sign of extravascular lung water. Am J Cardiol 2004, 93:1265-70. | Article | PubMed

9. Noble VE, Murray AF, Capp R, Sylvia-Reardon MH, Steele DJ and Liteplo $A$ : Ultrasound assessment for extravascular lung water in patients undergoing hemodialysis. Time course for resolution. Chest 2009, 135:1433-9. | Article | PubMed

10. Gargani L: Lung ultrasound: a new tool for the cardiologist. Cardiovasc Ultrasound 2011, 9:6. | Article | PubMed Abstract | PubMed Full Text

11. Sakka SG, Klein M, Reinhart K and Meier-Hellmann A: Prognostic value of extravascular lung water in critically ill patients. Chest 2002, 122:2080-6. | Article | PubMed

12. Volpicelli G, Caramello V, Cardinale L, Mussa A, Bar F and Frascisco MF: Bedside ultrasound of the lung for the monitoring of acute decompensated heart failure. Am J Emerg Med 2008, 26:585-91. | Article | PubMed

13. Gargani L, Lionetti V, Di Cristofano C, Bevilacqua G, Recchia FA and Picano E: Early detection of acute lung injury uncoupled to hypoxemia in pigs using ultrasound lung comets. Crit Care Med 2007, 35:2769-74. | Article | PubMed
14. Jambrik Z, Gargani L, Adamicza A, Kaszaki J, Varga A, Forster T, Boros $\mathrm{M}$ and Picano $\mathrm{E}$ : B-lines quantify the lung water content: a lung ultrasound versus lung gravimetry study in acute lung injury. Ultrasound Med Biol 2010, 36:2004-10. | Article | PubMed

15. Steen S, Ingemansson R, Budrikis A, Bolys R, Roscher R and Sjoberg T: Successful transplantation of lungs topically cooled in the non-heartbeating donor for 6 hours. Ann Thorac Surg 1997, 63:345-51. | Article | PubMed

16. Steen S, Sjoberg T, Pierre L, Liao Q, Eriksson L, Algotsson L. Transplantation of the heart from a non-beating donor. Lancet 2001, 357: 825-29.

17. D'Armini AM, Roberts CS, Griffith PK, Lemasters JJ and Egan TM: When does the lung die? I. Histochemical evidence of pulmonary viability after "death". J Heart Lung Transplant 1994, 13:741-7. | Article | PubMed

18. Webb WR: Thin-section CT of the secondary pulmonary lobule: anatomy and the image-the 2004 Fleischner lecture. Radiology 2006, 239:322-38. | Article | PubMed

\section{Citation:}

Wojtczak JA and Wood RW: High-frequency ultrasound in ex vivo animal lungs in pulmonary edema. journal of Anesthesiology and Clinical Science 2013, 2:21. http://dx.doi.org/10.7243/2049-9752-2-21 